\title{
Association of myelinated primary afferents impairment with mechanical allodynia in diabetic peripheral neuropathy: an experimental study in rats
}

\author{
Chenlong Liao ${ }^{1}$, Min Yang ${ }^{1}$, Wenxiang Zhong ${ }^{1}$, Pengfei Liu ${ }^{1}$ and Wenchuan Zhang ${ }^{1}$ \\ ${ }^{1}$ Department of Neurosurgery, Xinhua Hospital Affiliated to Shanghai JiaoTong University School of Medicine, Shanghai, \\ P. R. China \\ Correspondence to: Wenchuan Zhang, email: zhangwenchuan@xinhuamed.com.cn \\ Keywords: diabetic peripheral neuropathy, nerve decompression, mechanical allodynia, neuropathic pain \\ Abbreviations: DPN: diabetic peripheral neuropathy; MA: mechanical allodynia; PWL: paw withdrawal latency; PWT: paw \\ withdrawal threshold; STZ: streptozotocin \\ Received: April 05, $2017 \quad$ Accepted: June 16, $2017 \quad$ Published: July 18, 2017 \\ Copyright: Liao et al. This is an open-access article distributed under the terms of the Creative Commons Attribution License 3.0 \\ (CC BY 3.0), which permits unrestricted use, distribution, and reproduction in any medium, provided the original author and source \\ are credited.
}

\section{ABSTRACT}

To investigate the mechanisms underlying the efficacy of surgical treatment for painful diabetic peripheral neuropathy. Rats were initially divided into $\mathbf{3}$ groups (I, control rats, II, streptozotocin-induced diabetic rats, III, streptozotocin-induced diabetic rats with latex tube encircling the sciatic nerve without compression). When mechanical allodynia (MA) became stable in the third week, one third of group III rats were sacrificed and the remainder were further divided into subgroups depending on whether the latex tube was removed. Except for some rats in group III, all rats were sacrificed in the fifth week. Morphometric analysis of nerve fibers was performed. Expression level of $\mathrm{GABA}_{\mathrm{B}}$ receptor protein in spinal dorsal horn was determined. Changes of GABA $A_{B}$ receptor within areas of primary afferents central terminal were identified. Chronic nerve compression caused by the interaction of diabetic nerves swelling and the encircling latex tube increased the incidence of MA in diabetic rats, and nerve decompression could ameliorate MA. In diabetic rats with MA, demyelination of myelinated fibers was noted and reduction of $\mathrm{GABA}_{B}$ receptor was mainly detected in the area of myelinated afferent central terminals. MA in DPN should be partially attributed to compression impairment of myelinated afferents, supporting the rationale for surgical decompression.

\section{INTRODUCTION}

As a hallmark of neuropathic pain, mechanical allodynia (MA) is not uncommon in patients with painful diabetic peripheral neuropathy (DPN) [1], posing multidisciplinary therapeutic challenges. The outcome of pharmacological treatment is far from satisfactory due to limited efficacy and high rate of side effects [2]. As an alternate treatment, surgical decompression of multiple peripheral nerves [3] based on the "double crush" hypothesis [4] was proposed and has proven to be effective in relieving pain $[5,6]$. It is well accepted that diabetic neuropathic pain is caused by peripheral neuropathy [7] and a series of peripheral mechanisms were reported to underlie the development of painful DPN. Central changes, on the other hand, have also been considered as potential mechanisms $[2,8,9]$. Therefore, the effect of surgical decompression of peripheral nerves has been challenged and the mechanisms underlying the efficacy of surgical treatment for painful DPN remained uncertain and controversial. Ideally, both peripheral (input) and central (transmission) mechanisms should be considered together to provide a comprehensive understanding of underlying pathological processes and to develop better therapeutic strategies.

MA is well accepted to be mediated largely by myelinated $A \beta$-fibers [10]. Abnormal $A \beta$ sensory input is 
reported to play an important role in diabetic neuropathic pain [7]. According to the gate control theory of pain, $[11,12]$ input of the myelinated $A \beta$-fibers is gated by feedforward activation of inhibitory neurons $[11,12]$ Hence, as suggested by central mechanisms, MA might be attributed to imbalance between excitatory (mostly glutamate) and inhibitory (mostly GABA and glycine) neurotransmitters in the spinal cord $[8,13]$.

The $\mathrm{GABA}_{\mathrm{B}}$ receptor is widely distributed in the central nervous system including the spinal cord [14], and serves as autoreceptor for feedback regulation of synaptic GABA release as well as heteroreceptors that regulate synaptic glycine and glutamate release to the spinal dorsal horn neurons [15-17]. It is hypothesized that downregulation of presynaptic $\mathrm{GABA}_{\mathrm{B}}$ receptor at afferent terminal is involved in diabetic neuropathic pain by increasing glutamatergic input to the spinal dorsal horn neurons [18].

In the light of previous clinical and basic research, the present study was conducted with the intention to investigate the mechanisms accounting for the efficacy of surgical decompression of peripheral nerves for painful DPN.

\section{RESULTS}

\section{Rat phenotype after streptozotocin injection}

The rats $(n=3)$ that died or failed to develop hyperglycemia following streptozotocin (STZ) injection were excluded from this study (not shown in Figure 1). 10 rats in group I served as control group (group 1). 20 rats in group II and 30 rats in group III developed hyperglycemia (blood glucose $>350 \mathrm{mg} / \mathrm{dL}$ ) and displayed polyuria, a reduced growth rate and a marked increase in food and water intake throughout the experimental period, but otherwise the rats remained relatively healthy. Different degrees of nerve swelling, which render the diabetic nerve susceptible to chronic compression by the latex tube, could be noted in all diabetic rats during the nerve harvest procedures at the time of sacrifice. During the surgical procedure, the site of ischial notch where the sciatic nerve passes through was identified as a potential compression site for diabetic swelling nerve since it is quite narrow in nature.

\section{Mechanical compression increased the incidence of mechanical allodynia in diabetic rats}

The results of behavioral tests were shown in Figure 2. After 3 weeks, 11 rats in group II, without latex tube enclosure, and 26 rats in group III developed persistent MA. Eight rats in group III, with latex tube encirclement, were sacrificed in the third week (group 3) while the other 18 group III rats were further randomly and evenly divided into two groups, becoming Groups 4 and 5, which had latex tube removal or retention. More diabetic rats with latex tube surrounding the sciatic nerve developed MA (26/30) than those with sham operation $(11 / 20, P=0.012<0.05)$.

The average paw withdrawal threshold (PWT), measured with von Frey filaments, were significantly lower in both group II $(4.35 \pm 0.19)$ and group III $(4.34 \pm 0.13)$ two weeks after STZ injection when compared with that in control group $(13.743 \pm 0.43, P<0.05, P<0.05)$. In the third week, no significant difference on PWT was noted between group II and III $(P>0.05)$. Meanwhile, as regard to the subgroup 4 and 5, no significant difference on PWT was noted $(P>0.05)$. By the fifth week, recovery of higher PWT was observed in group 4 with tube compression removal when compared with that in group $5(P<0.05)$ (Figure 2A).

\section{Mechanical compression did not increase the incidence of thermal hyperalgesia in diabetic rats}

Most rats in group II (19/20) and III (28/30) developed persistent thermal hyperalgesia at 3 weeks after induction of diabetes. The paw withdrawal latency (PWL) of control group is higher than any experimental group $(P<0.05)$. No significant difference in PWL was noted among the experimental groups 2,3,4 and 5 during the experimental period $(P>0.05)$ (Figure 2B).

\section{Varying degrees of demyelination of myelinated A-fibers was observed in diabetic rats with mechanical allodynia}

Compared with the control group, an increased degree of demyelination of myelinated fibers was observed in all the experimental groups (Figure 3). As summarized in Table 1, more reduction in myelinated fiber area and density were noted in all the experimental groups when compared with the control group $(P<0.05)$. Both myelinated fiber area and density in group 3 were larger than those in group 5 with more prolonged compression (3rd week vs 5th week). Myelinated fiber area and density were larger in group 4 than those in group 2 and $5(P<0.05)$. Correspondingly, higher g-ratio (axon area/ fiber area), which is an indication of demyelination, was noted in all the experimental groups when compared with the control group $(P<0.05)$. G-ratio was higher in group 3 than that in group 5. When compared with group 2 and 5, higher g-ratio was noted in group 4. On the other side, degeneration of unmyelinated axons, demonstrated by the morphological changes, could be noted in all the experimental groups. While higher unmyelinated axons area and density was observed in the control group than all experimental groups $(P<0.05)$, no significant difference in unmyelinated axon area and density were noted among the four experimental groups $(P>0.05)$. 
Downregulation of $\mathrm{GABA}_{\mathrm{B}}$ receptor was found in all the diabetic rats with mechanical allodynia and was mainly detected in the area of myelinated afferent terminals

Lower expression level of $\mathrm{GABA}_{\mathrm{B}}$ receptor protein was found in all experimental groups compared to the control group $(P<0.05)$. Although it remained at a low level, expression of $\mathrm{GABA}_{\mathrm{B}}$ receptor protein in group 4 was higher than that in group $5(P<0.05)$ (Figure 4).

Figure 5 shows the immunofluorescence outcome in the spinal dorsal horn of all groups. While fluorescence of $\mathrm{GABA}_{B}$ receptor in the areas of NF$200^{+}$neurons, albeit faint, could still be observed in the 4 experimental groups, it could hardly be noted in the areas of NF- $200^{+}$myelinated afferent terminals

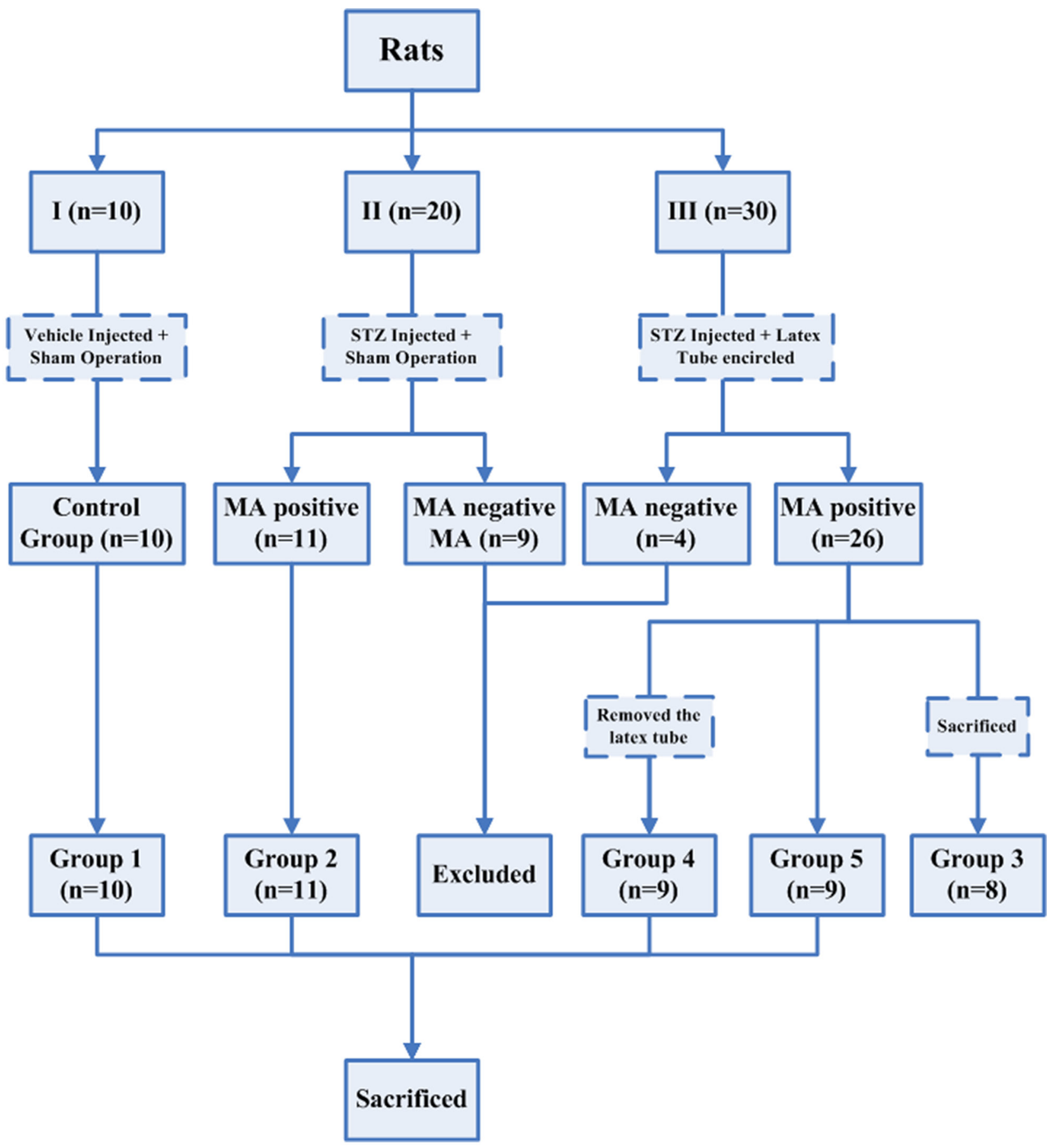

Figure 1: Flowsheet showing different groups of rats that were subjected to different treatment throughout the experimental period. 
(Figure 5A). On the other hand, immunofluorescence staining of $\mathrm{GABA}_{\mathrm{B}}$ receptor could be observed in both areas of $\mathrm{CGRP}^{+}$neurons and $\mathrm{CGRP}^{+}$unmyelinated afferent terminals in all groups (Figure 5B). Figure 5C demonstrated the area percentages of double-labeled central terminals of primary afferents by $\mathrm{GABA}_{B}$ receptor and NF-200 or CGRP in all the area of $\mathrm{GABA}_{B}$ receptor labeled primary afferents terminals. The area of double-labeled primary afferents terminals by $\mathrm{GABA}_{\mathrm{B}}$ Receptor and NF-200 was larger in the control group compared to the other 4 experimental groups $(P<0.05)$. On the contrary, no significant difference in double-labeled primary afferent terminal area was noted among all the experimental groups.

A

\section{Tests for Mechanical Allodynia}

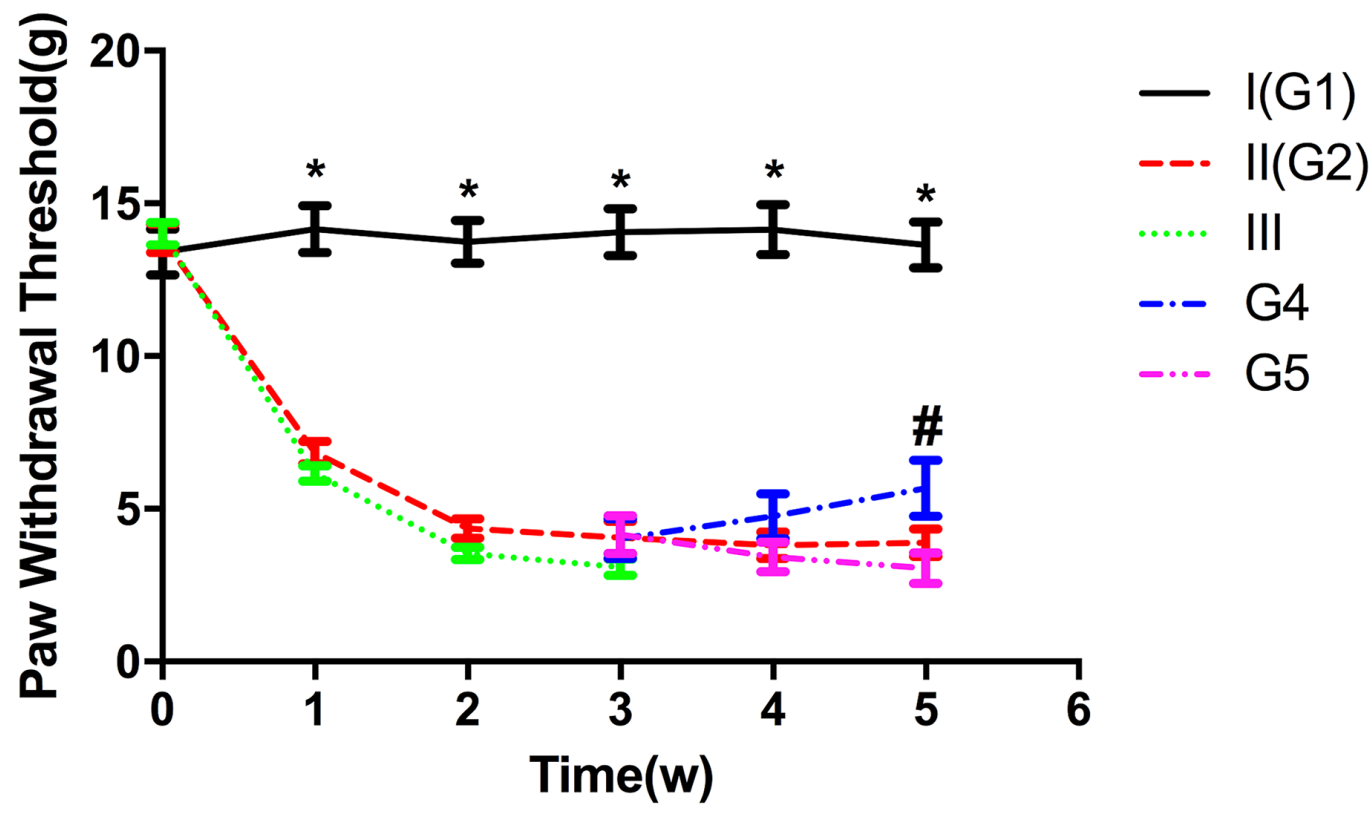

B

Tests for Thermal Hypergesia

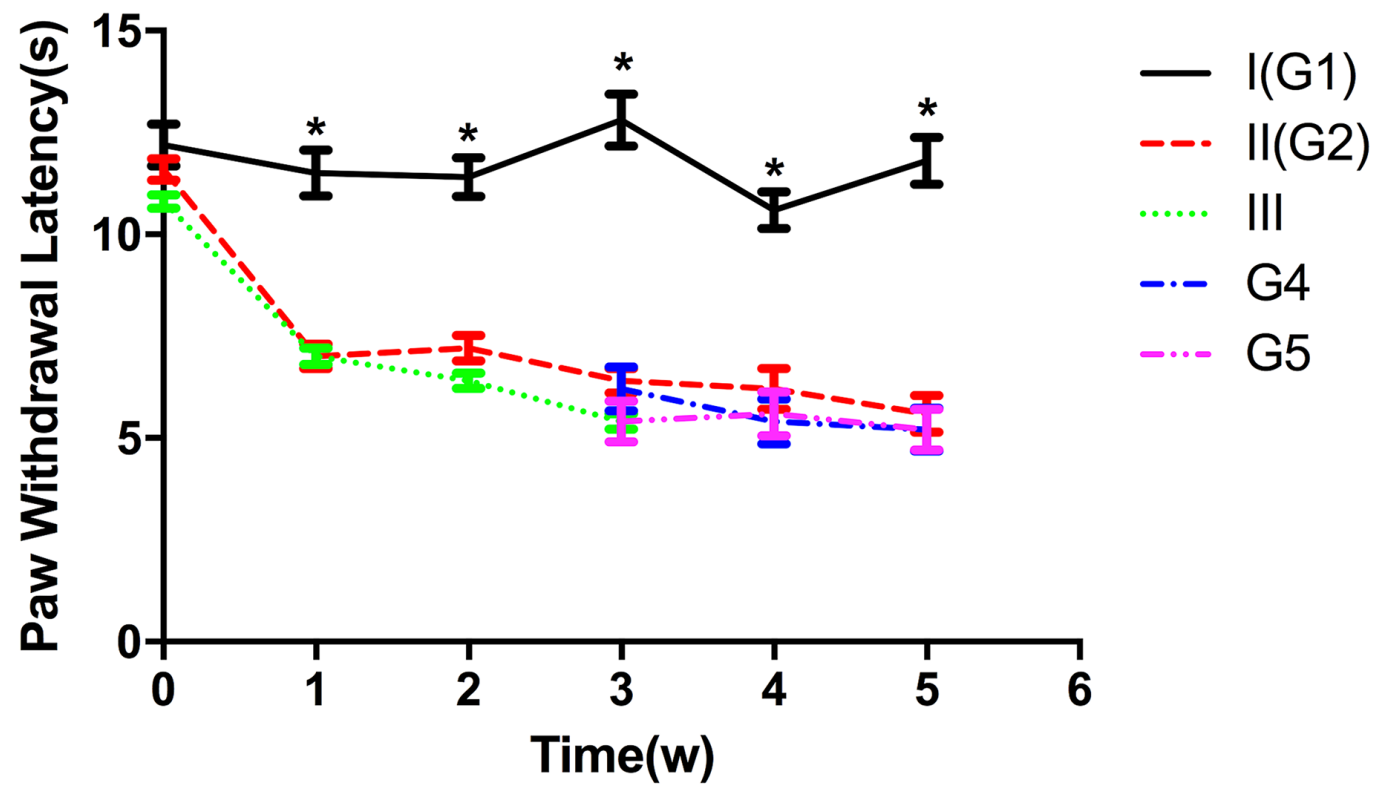

Figure 2: Diagram of behavioral tests showing the changes in the paw withdrawal thresholds (A) and paw withdrawal latency (B) in all groups. ${ }^{*} P<0.05$ compared with respective experimental groups values. $\# P<0.05$, group 4 versus group 5 . 
Table 1: Morphometric data of both myelinated and unmyelinated fibers in all groups

\begin{tabular}{ccccccc}
\hline Groups & $\begin{array}{c}\text { MF density } \\
\left(\mathbf{n u m b e r} / \mathbf{m m}^{2}\right)\end{array}$ & $\begin{array}{c}\text { MF axon area } \\
\left(\boldsymbol{\mu m}^{2}\right)\end{array}$ & $\begin{array}{c}\text { MF fiber area } \\
\left(\boldsymbol{\mu m}^{2}\right)\end{array}$ & MF g-ratio & UA area $(\boldsymbol{\mu m})$ & $\begin{array}{c}\text { UA density } \\
\left(\mathbf{n u m b e r} / \mathbf{m m}^{2}\right)\end{array}$ \\
\hline 1 & $7863 \pm 162^{\#}$ & $26.83 \pm 2.74$ & $67.4 \pm 3.4^{\#}$ & $0.54 \pm 0.09^{\#}$ & $0.76 \pm 0.04^{\#}$ & $8,2637 \pm 5372^{\#}$ \\
2 & $4045 \pm 157$ & $25.26 \pm 2.33$ & $42.8 \pm 2.7$ & $0.66 \pm 0.15$ & $0.46 \pm 0.07$ & $5,7382 \pm 4726$ \\
3 & $4828 \pm 168^{*}$ & $25.65 \pm 2.16$ & $45.2 \pm 2.5^{*}$ & $0.68 \pm 0.14^{*}$ & $0.47 \pm 0.04$ & $6,0328 \pm 4636$ \\
4 & $4652 \pm 188^{\star \star}$ & $26.42 \pm 2.08$ & $49.3 \pm 2.3^{\dagger}$ & $0.60 \pm 0.11^{\dagger}$ & $0.44 \pm 0.03$ & $5,3804 \pm 5022$ \\
5 & $3870 \pm 211$ & $24.68 \pm 2.24$ & $39.6 \pm 2.1$ & $0.75 \pm 0.23$ & $0.42 \pm 0.04$ & $5,0173 \pm 4883$ \\
\hline
\end{tabular}

Data are expressed as mean \pm SEM. MF, myelinated fiber; UA, unmyelinated axon.

${ }^{\#} P<\mathbf{0} .05$ Group 1 versus group $2-5 ;{ }^{*} \mathrm{P}<0.05$ Group 3 versus group $5 ; \uparrow \mathrm{P}<0.05$ Group 4 versus group $5 ; \ddagger \mathrm{P}<0.05$ Group 4 versus group

\section{DISCUSSION}

Since peripheral nerves in diabetes are proven to be susceptible to mechanical compression [19], a modified rat model of chronic nerve compression, in which the diabetic sciatic nerve distal to the ischial notch was surrounded by a $1.5-\mathrm{cm}$ length of latex tube $[20,21]$, was used for this study. Nerve trunk swelling as diabetes is induced exposes the rat nerve to progressive chronic compression by the encircling latex tube, as occurs in human diabetes at known sites of fibro-osseous anatomic narrowings, such as carpal, cubital and tarsal tunnels [22].

In the present study we have found that chronic nerve compression increases the incidence of $\mathrm{MA}$ in diabetic rats (group II V.S. III) and in turn, nerve decompression after 2 weeks of hyperglycemia decreases MA as measured by PWT (group 4 vs 5). These data verified that MA in DPN does indeed result, at least partly, from nerve compression. Other salient findings are that demyelination of myelinated fibers was observed in diabetic rats with MA, and that downregulation of $\mathrm{GABA}_{\mathrm{B}}$ receptor, reported previously to be involved in the development of MA in DPN [18], was mainly detected in the area of myelinated afferent terminals in spinal cord dorsal horn. Combining the morphological impairments in peripheral nerve fibers with the molecular changes in central terminals, we hereby suggest that MA in DPN is associated with structural and functional alteration of myelinated afferent fibers. Consistent with the prior understanding that large myelinated A-fibers are more sensitive to mechanical compression than small unmyelinated $\mathrm{C}$ fibers [23, 24], these findings of the present study strongly imply that myelinated A-afferent fibers, rather than unmyelinated $\mathrm{C}$-afferent fibers, are the axons impaired by chronic nerve compression in DPN and lead to the development of MA. Accordingly, the rationale of surgical decompression for painful DPN is vindicated and the reported benefit explained.

Confirming previous studies [25], this report demonstrates that demyelination and degeneration of nerve fibers are prominent features in DPN, resulting in increased g-ratio of axon area/fiber area, and reduction in myelinated fiber area, and density of both myelinated and unmyelinated fibers. Myelinated fiber impairment, we attributed largely to the compression since decompression of the nerve would delay and/or restore the impairment, as shown by larger myelinated fiber area and density in group 4 than in group 5. During the surgical procedure, we
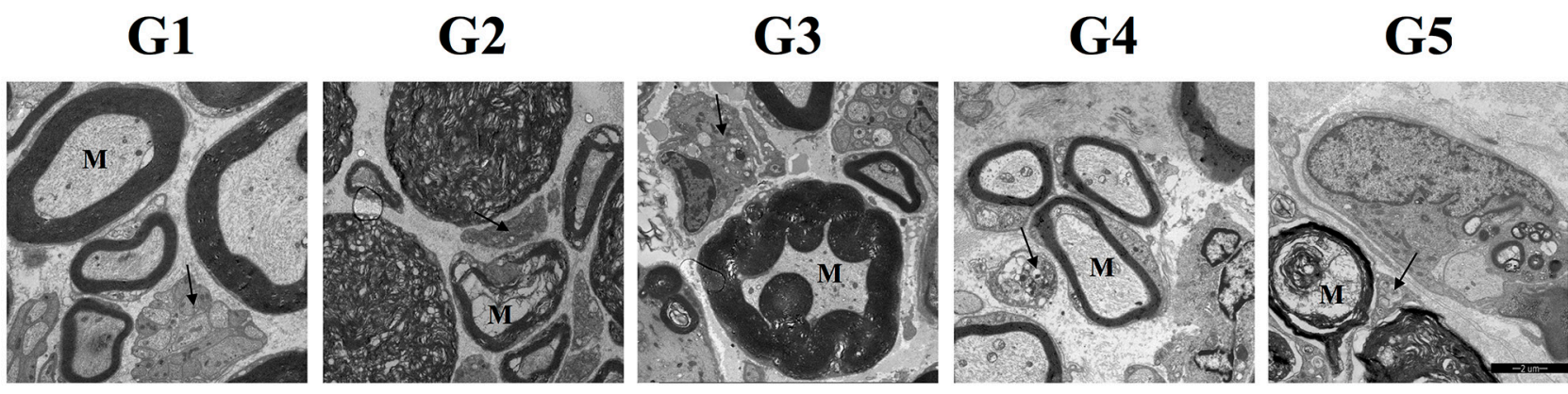

Figure 3: Electron micrographs of sciatic nerves in all groups. The black arrows in micrographs point to the unmyelinated fibers and the letter " $\mathrm{M}$ " indicates myelinated fibers. Demyelination and degeneration of myelinated fibers and unmyelinated fibers could be noted in the 4 experimental groups. Abnormal morphological structures of unmyelinated fibers, manifesting as dense and collapse configuration, and different degree of myelin impairment could be noted in the experimental groups. 
identified the site of ischial notch as one of the potential anatomic compression sites in rats of group 2. A swelling sciatic nerve could be compressed here in the setting of diabetes, causing pain symptom. This may also explain why would diabetic rats without latex tube surrounding the sciatic nerve still develop painful DPN. In this way, the result that not all the rats develop painful DPN may owe to the anatomic distinction that not all the diabetic nerve would be compressed by the ischial anatomic narrowing. It might be similar in clinical practice.

Both myelinated fiber area and density in group 3 by the 3 rd week sacrifice were larger than those in group 5 at 5 weeks, so impairment of myelinated fibers in painful diabetic rats appears time-dependent. Therefore, patients with painful DPN would benefit most from surgical decompression in an early stage. Early surgery is also supported by several previous clinical reports [26-28].

In addition to MA a, thermal hyperalgesia was likewise induced in most of the diabetic rats. Morphological changes of unmyelinated C-fibers, the main thermal nociceptor [29], could be observed in all groups (Figure 3). However, these changes might not be attributed to mechanical compression since unmyelinated C-fibers are more vulnerable to inflammatory injury [24],
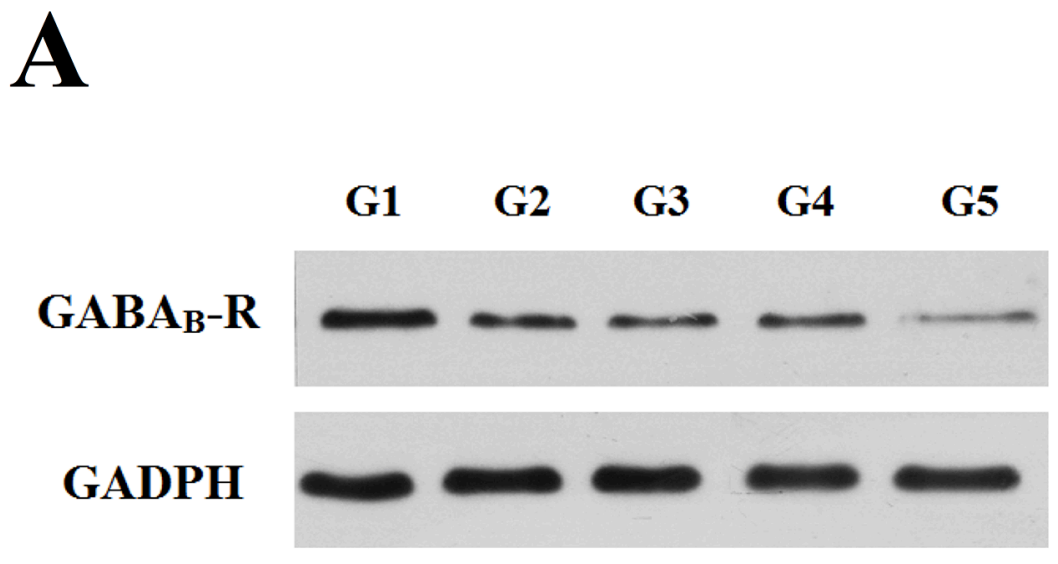

B

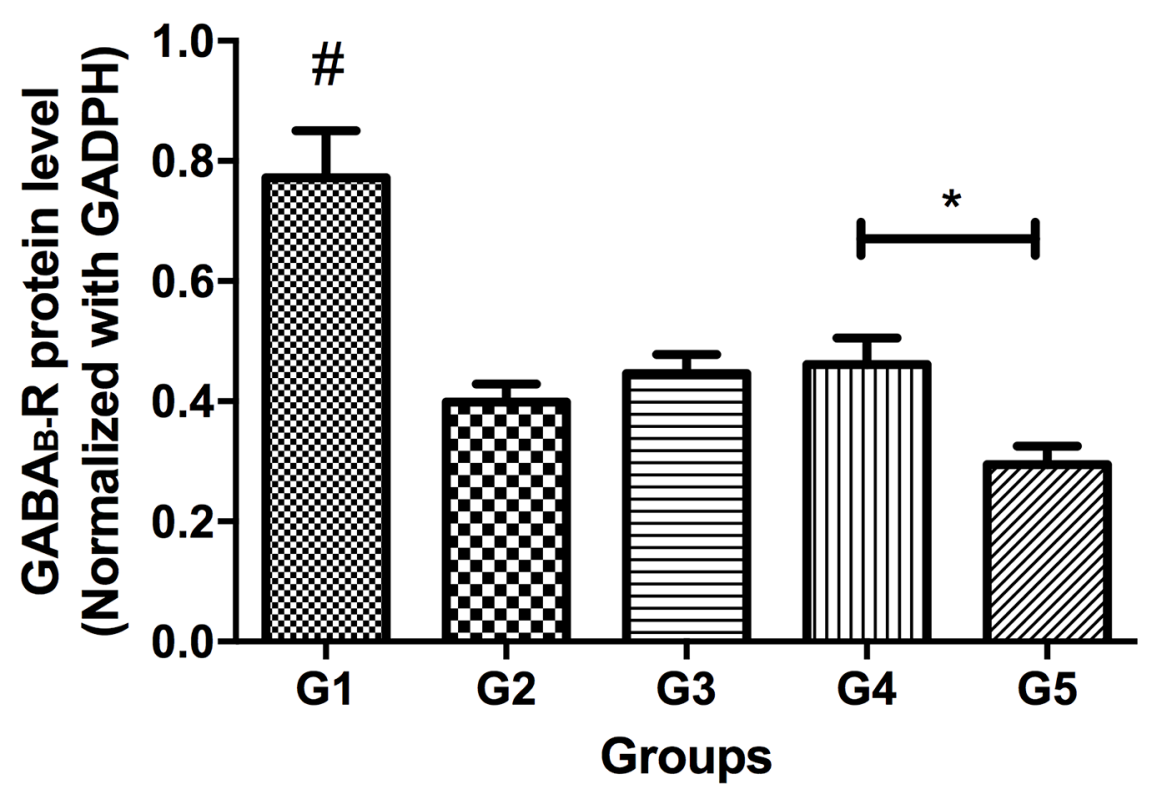

Figure 4: Western blot analysis of $\mathrm{GABA}_{\mathrm{B}}$ receptor protein in spinal cord (L5) in all groups. (A) Representative bands, (B) summary data. $* P<0.05$, group 4 versus group 5 . \#, control group versus experimental groups. 
which is common in diabetes [30]. This interpretation is supported by our finding that mechanical compression did not increase the incidence of thermal hyperalgesia in diabetic rats, and that decompression ameliorated the degree of demyelination of myelinated axons but not the morphological changes of unmyelinated axons.

In the present study we investigated both peripheral (primary afferent fibers) and central (spinal dorsal horn)

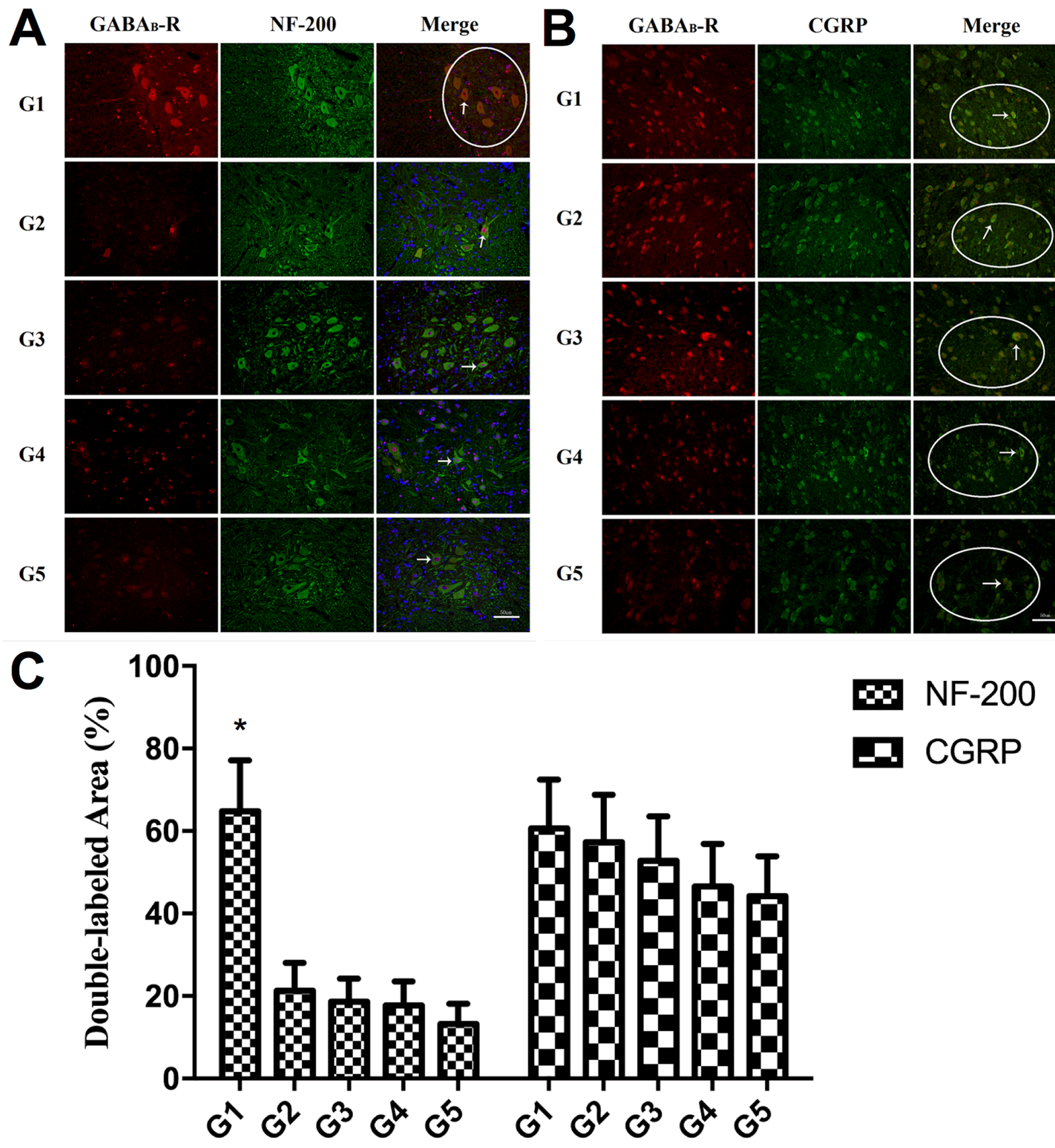

Figure 5: (A) Double immunofluorescence staining of GABAB receptor protein and NF-200 in the spinal dorsal horn (L5). Fluorescence of $\mathrm{GABA}_{\mathrm{B}}$ receptor in the areas of $\mathrm{NF}-200^{+}$neurons can be observed in all the groups (white arrow). In contrast to the control group, in which fluorescence of $\mathrm{GABA}_{\mathrm{B}}$ receptor can be noted in the areas of NF-200 myelinated afferent terminals (white circle area), little fluorescence staining of $\mathrm{GABA}_{\mathrm{B}}$ receptor can be seen in the areas of NF- $200^{+}$myelinated afferent terminals in the 4 experimental groups. Scale bar: 50um. (B) Double immunofluorescence staining of GABA receptor protein and CGRP in the spinal dorsal horn (L5). Immunofluorescence staining of $\mathrm{GABA}_{\mathrm{B}}$ receptor could be observed in both areas of $\mathrm{CGRP}^{+}$neurons (white arrow) and CGRP ${ }^{+}$ unmyelinated afferent terminals (white circle area) in all groups. Scale bar: 50um. (C) The histogram showing the area percentages of double-labeled primary afferents terminals by $\mathrm{GABA}_{\mathrm{B}}$ Receptor and NF-200 or CGRP in all the GABA $\mathrm{B}_{\mathrm{B}}$ Receptor labeled primary afferents terminals. * $P<0.05$, control group versus group $2-5$. 
changes of nervous systems in rat DPN. Our results suggest that it is the myelinated A-afferent, rather than unmyelinated $\mathrm{C}$-fibers, which are involved in the development of MA in DPN. This is consistent with the results from the Kahn et al functional and structural study on the important role of myelinated afferent nerves in allodynia caused by diabetic neuropathy [7]. Although MA represents a cardinal feature of neuropathic pain, it is only one variety of pain symptom. Patients with DPN describe several other pain types such as aching, burning, painful tingling, lancinating pains and hyperalgesia that can seriously reduce their quality of life [31]. While spontaneous pain has been variously described as burning, shooting, lancinating or crawling in character [2], the evoked pains, including allodynia and hyperalgesia, are often further distinguished according to the different sensory modalities (touch, pressure, pinprick, vibration, cold, heat) that are used to elicit them [32].

Also, it is still a clinical conundrum that why some patients with DPN experience distressing pain while others develop the insensate symptom numbness [33]. Although the natural course of painful diabetic neuropathy is variable [34] and neuropathic pain could be present at any stage of DPN [35], it was suggested that positive symptoms, such as pain and hyperalgesia, is generally present in early stage of DPN, whereas advanced stage is typically characterized by insensate symptoms, such as mechanical and thermal hypoalgesia [36-38]. This time-dependent behavioral switch should be attributed to the consequence of different degree of nerve injury that may range from active degeneration or impaired regeneration to loss of sensory fibers [39]. Although multiple metabolic mechanisms were reported to participated in this process [40-45], demyelination caused by mechanical compression shown in the present study should not be ignored. The evidence comes from the comparison between models of STZ-diabetic rats and mice. STZdiabetic rats were reported to develop thermal and mechanical hyperalgesia within 2-8 weeks and become hypoalgesic after longer periods of diabetes [40, 46, 47] while hyposensitivity to heat and mechanical stimuli, rather than hyperalgesia and MA, was found in STZ-treated mice [48-50]. Correspondingly, in early stage, signs of demyelination could be observed in STZ-diabetic rats as shown in the present study and other previous reports $[51,52]$, but not in diabetic mice [53]. Nevertheless, neuropathic pain can be induced in several nerve compression models in mice, such as chronic constriction injury model, spinal nerve ligation model and spared nerve injury model [54-56], suggesting that diabetic nerve in rats is compromised by both metabolic inflammation and mechanical compression while that in diabetic mice may be impaired by solely metabolic inflammation.
This species-dependent difference on positive and negative symptoms may result from either distinction of anatomic narrowings or the different susceptibility of the nerves to compression in the setting of DPN.

The diverse characteristics of pain symptoms could imply that a number of mechanisms are involved, with various patients (even in each stage of DPN) having a differing pathogenic profile. However, mechanisms of neuropathic pain are neither disease-specific nor symptomatic-specific $[57,58]$. As classified by Spallone et al., in the case of peripheral neuropathic pain, damage to peripheral sensory nerves and subsequent primary afferent activity was considered as the initial event, with the manifestations of pain symptoms resulting from consequent changes in structure and function of the somatosensory nervous system [59]. This may be the reason why large numbers of previous studies failed to identify a significant functional or pathological abnormality related to pain in diabetic neuropathy $[60,61]$.

Making a distinction between spontaneous pain and evoked pain is difficult in both clinic work and laboratory research. According to the Jensen and Finnerup, "it would be impossible to establish whether a stimulus is capable of activating nociceptors in the individual patient" [32] and in turn, patients may take provoked pain as spontaneous pain in daily life and thus provide inaccurate information about the characteristic of pain. In parallel, it is not possible to quantify spontaneous pain in animals [33] and reduced PWT or PWL is frequently presumed as a general pain symptom in many models of pain. Therefore, while we have demonstrated surgical decompression is effective in ameliorating MA, we also show that decompression is not effective for all types of DPN pain. It seems appropriate to view DPN as a heterogenous disease state, and other mechanism-based multimodal treatments should be further investigated.

Sciatic nerve compression in streptozotocininduced diabetic rats produces mechanical allodynia and thermal hyperalgesia manifest in paw withdrawal. These objective behavioral changes correlate with sciatic nerve axonal $A \beta$-fiber demyelination and degeneration of unmyelinated axons. Downregulation of GABAB receptor was present in all diabetic rats with mechanical allodynia in the spinal cord areas of myelinated afferent terminals. Surgical decompression by removal of a sciatic encircling latex tube reduced allodynia but not thermal hyperalgesia.

A role of compression impairment of myelinated afferents in producing allodynia and the benefit of decompression is confirmed, revealing the rationale of surgical decompression. This demonstrated amelioration of allodynia provides justification for clinical use of external neurolysis of fibro-osseous tunnel compression sites in painful human diabetic peripheral neuropathy. 


\section{MATERIALS AND METHODS}

\section{Study approval}

All procedures involving animals were in accordance with the guideline for the Care and Use of Laboratory Animals. This study protocol was approved by Ethics Committee of Xinhua Hospital (NO. XHEC-F-2016-009). All efforts were made to minimize both the suffering and number of animals.

\section{Animals and experimental design}

Male Sprague-Dawley rats initially weighing 150$180 \mathrm{~g}$ were used. The rats were housed at a temperature of $21-24^{\circ} \mathrm{C}$ and maintained a $12-\mathrm{h}$ light/dark cycle throughout the experimental period. The rats were initially randomly divided into 3 groups according to the different treatments (Figure 1). Diabetes was induced in rats of groups II and III by a single intraperitoneal administration of STZ (60mg/kg; Sigma, St. Louis, MO, USA) freshly dissolve in $0.1 \mathrm{~mol} / \mathrm{L}$ citrate buffer $(\mathrm{pH} 4.5)$. Weight-matched vehicle (sodium citrate buffer) was injected into the rats of control group. Diabetes was confirmed three days later by measurement of plasma glucose concentration $(>350 \mathrm{mg} /$ $\mathrm{dL}$ ) in blood samples obtained from the tail vein. The glucose level was assayed enzymatically using Sigma diagnostic glucose reagents.

To test the hypothesis that the underlying metabolic abnormalities involved in diabetes render the peripheral nerve susceptible to chronic nerve compression, a modified rat model of chronic nerve compression was used in this study to investigate the effect of mechanical compression on the development of MA caused by DPN [20] All surgical procedures were performed under aseptic conditions by one surgeon (Liao). The rats were anesthetized with an intraperitoneal injection of sodium pentobarbital $(50 \mathrm{mg} / \mathrm{kg})$, followed by the induction of diabetes. The hair in the right thigh region was removed and the skin was sterilized with $0.5 \%$ povidone solution. The skin and muscle layer of the lateral surface of the right thigh was incised and the sciatic nerve was exposed. A $1.5 \mathrm{~cm}$-long latex tube was used to surround the sciatic nerve on the distal site of ischial notch. The tube was sutured with three sutures of 7-0 nylon, thereby encircling without compressing the nerve (the tube could be easily moved back and forth along the nerve). Muscle and skin layer was sutured with silk thread and topical antibiotic powder was applied at once.

This model of chronic nerve compression was built in rats of group III while sham operation was performed in rats of group I and II. The diabetic rats in group II and group III that did not develop MA within 3 weeks were excluded. Meanwhile, one-third rats with MA in group III were sacrificed (as group 3) at the end of the third week and the remainder were then further divided into two groups (as group 4 and 5). After 3 weeks, latex tube was removed in rats of group 4 but was retained in rats of group 5. Excluding the group 3 rats sacrificed in the third week, all other rats were sacrificed in the fifth week (Figure 1). The sample size and time points were based on the results of our preliminary experiments and the power analysis.

\section{Behavioral analysis}

MA and thermal hyperalgesia were tested weekly by two observers blinded to the experimental groups. The observers were not blind to control group and experimental groups due to the distinct physical appearance of diabetes.

MA was determined by quantifying the PWT in response to von Frey filaments (Stoelting, Wood Dale, USA). The rats were acclimated for 30 minutes in suspended individual chambers on a mesh floor. A series of calibrated von Frey filaments, starting from the lowest force filament, were applied perpendicularly to the plantar surface of the hindpaw for 6 seconds with sufficient force to bend the filament. Brisk withdrawal or paw licking was considered as a positive response. In the absence of a response, the filament of next greater force was applied. In the presence of a response, the filament of next lower force was applied. The mechanical stimulus producing a 50\% likelihood of withdrawal response was calculated by using the 'up-and-down' method as described previously [62, 63]. The lowest force filament able to produce a positive reaction is determined based on 3 positive responses in at least 5 repeated measurements. Each testing was performed at 2-min intervals to determine a mean value.

Thermal hyperalgesia was determined by PWL in response to radiant heat. Rats were placed within plexiglass enclosures on a transparent $30^{\circ} \mathrm{C}$ glass surface. After acclimatizing for 30 minutes, a thermal testing apparatus (IITC/Life Science, woodland Hills, CA, USA) was used to apply radiant noxious heat to the plantar surface of the hindpaw. The latency time to paw withdrawal was measured by a digital timer. Testing was repeated 3 times at 2-min intervals to determine a mean value for the thermal threshold. A no-response cutoff of 30 seconds was used to prevent potential tissue damage [64].

\section{Morphological study}

Before sacrifice, rats were anesthetized as described above and the right sciatic nerves distal to the ischial notch were quickly transected and separated into two segments. One was post-fixed overnight in $4 \%$ paraformaldehyde and dehydrated stepwise in increasing concentration of ethanol prior to embedding in paraffin. A $1.5 \mu \mathrm{m}$ transverse section was cut on a Leica RM2016 microtome, stained with toluidine blue and examined under a light microscope. The second was fixed in $2.5 \%$ glutaraldehyde solution. After 
24 hours, the tissues were transferred into phosphatebuffered saline $(\mathrm{pH} 7.4)$ and then were post-fixed in $1 \%$ osmium tetroxide for 2 hours, dehydrated stepwise in increasing concentration of ethanol followed by propylene oxide. The tissues were finally impregnated with a series of mixtures of resin and propylene oxide and embedded in $100 \%$ epoxy resin. The semithin sections $(<1 \mu \mathrm{m})$ were examined under an electron microscope.

The number and density of myelinated fibers was counted on the light microscope montages. Morphometric assessments were made on about 500 myelinated fibers in each specimen. Electron micrographs $(\times 3000)$ of at least 300 myelinated fibers were prepared for each specimen to calculated the myelinated axon and fiber area and g-ratio (axon area/fiber area). Electron micrographs $(\times 10$, 000 ) of at least 30 unmyelinated fibers were collected by a systematic random sampling technique [61]. The unmyelinated axons were identified with the use of established criteria, including the presence of neurotubules and neurofilaments defined from electron micrographs at high magnification and the circularity and distribution of the axons enveloped by the Schwann cell [65]. The number and diameter of unmyelinated axons were directly counted and the density was derived from the total endoneurial area assessed. Calculation was performed with the use of AnalySIS Pro 3.1 (Soft Imaging Systems $\mathrm{GmbH}$, Munster, Germany) by two observers who were blinded to the groups. All rats in each group were used in morphological assessments (Figure 1).

\section{Western blot}

The L5 spinal cord was transected on ice and the tissue was homogenized in protease inhibitor cocktail (Sigma, St. Louis, MO) and RIPA lysis buffer. An equal amount of protein $(60 \mu \mathrm{g})$ was loaded into each lane, separated electrophoretically by SDS-polyacrylamide gel and transferred to polyvinylidene fluoride membranes. After washing in TBS with $0.1 \%$ Tween-20 (TBS-tween), the membranes were blocked with $5 \%$ nonfat milk dissolved in TBS-tween for 1 hour at room temperature and incubated overnight at $4{ }^{\circ} \mathrm{C}$ with rabbit monoclonal anti- $\mathrm{GABA}_{\mathrm{B}}$ Receptor 1 antibody (1:500, Abcam, catalog NO. ab55051) and anti-GADPH (1:1000, Wuhan goodbio technology, Wuhan, China). After several washes in TBStween, the membranes were incubated with horseradish peroxidase-conjugated goat anti-rabbit secondary antibody (1:3000, Wuhan goodbio technology, Wuhan, China) for 1 hour at room temperature. Finally the membranes were washed in TBS-tween and chemiluminescent bands were detected using ECL technology (Wuhan goodbio technology, Wuhan, China; G2014). The intensity of the bands was captured digitally and analyzed quantitatively by a blinded observer using the alphaEaseFC (Alpha Innotech) and Adobe PhotoShop (Adobe). All GABA receptor protein concentrations were normalized to
GADPH protein. Specimens from half number of the animals in each group were used in western blot.

\section{Immunofluorescence}

Under deep anesthesia, rats were then transcardially perfused with $150 \mathrm{ml}$ of saline followed by $250 \mathrm{ml}$ of $4 \%$ paraformaldehyde in $0.1 \mathrm{M}$ phosphate-buffered saline (pH 7.4). The L5 spinal cord was quickly transected and postfixed by immersion in the same fixative solution overnight. The spinal cord tissue was dehydrated, embedded in paraffin and sectioned at a thickness of $5 \mu \mathrm{m}$. The sections were preincubated in blocking solution ( $10 \%$ goat serum, $1 \%$ Triton X-100 in PBS) for 30 minutes at room temperature, followed by overnight incubation at $4^{\circ} \mathrm{C}$ with rabbit monoclonal anti-GABA ${ }_{\mathrm{B}}$ Receptor 1 antibody (1:400, Abcam, catalog NO. ab55051) and either mouse monoclonal anti-Hypophosphorylated neurofilament $\mathrm{H}$ antibody (anti-NF-200, a marker for myelinated A-fibers [66], 1:200, Abcam, catalog NO. ab82259) or mouse monoclonal anti-Calcitonin gene related peptide antibody (anti-CGRP, a marker for unmyelinated C-fibers [67], 1:100, Abcam, catalog NO. ab81887) in blocking solution. After three washes with PBS, the fluorophoreconjugated secondary antibodies, including Alexa Fluor 647 AffiniPure Goat Anti-Rabbit IgG antibody (1:100, Jackson) and FITC-AffiniPure Goat Anti-mouse IgG antibody (1:50, Jackson) were applied for 1 hours at room temperature. Cell nuclei were counterstained with DAPI. Control experiments determining the specificity of the immunofluorescence were performed by omitting the primary antibodies. The stained sections were examined with the Olympus FluoView 1000 confocal laser scanning microscope. In spinal dorsal horn, the areas of central terminals of primary afferents that were double labeled with $\mathrm{GABA}_{\mathrm{B}}$ Receptor and NF-200 or CGRP were calculated to detect the changes of presynaptic $\mathrm{GABA}_{\mathrm{B}}$ receptor level within the central terminals of two different primary afferents with the use of Image Pro plus 6.0 (Media Cybernetics, Silverspring, USA). Specimens from half number of the animals in each group were used in immunofluorescence assay, which was performed on 5 sections from each animal. Observation was performed within the area of laminae I-V and at least 5 areas of each section were evaluated.

\section{Statistical analysis}

Comparison between the number of rats developing MA and thermal hyperalgesia in different groups were analyzed with the Pearson's $\chi^{2}$ test. The quantification of protein expression, fluorescence staining and the morphometric analyses were performed with the use of the one-way ANOVA followed by Tukey's post-hoc test. Repeated measurement Two-way ANOVA with Tukey's post-hoc test was used to determine the levels of blood 
glucose, changes of PWT and PWL in different groups along the experimental period. All the data were expressed as mean \pm SEM. Statistical analyses were completed with SPSS 18.0. The criterion for statistical significance was $P<0.05$.

\section{Author contributions}

C.L.L. designed the study and prepared the manuscript. C.L.L., P.F.L. and W.X.Z. contributed to data analysis and performed the experiments. M.Y. contributed to data analysis. W.C.Z. supervised the overall study design and contributed significantly to the manuscript. All authors reviewed the manuscript.

\section{ACKNOWLEDGMENTS}

We present our sincere gratitude to Professor D. Scott Nickerson (Northeast Wyoming Wound Care Center, Sheridan Memorial Hospital) for his careful and patient copy-edit work and review on this manuscript.

\section{CONFLICTS OF INTEREST}

The authors declare that they have no conflicts of interest.

\section{FUNDING}

This work was supported by the National Science Foundation of China (NO. 81371373) and MedicalEngineer cross fund of Shanghai JiaoTong University (No. YG2014QN18).

\section{REFERENCES}

1. Baron R, Tolle TR, Gockel U, Brosz M, Freynhagen R. A cross-sectional cohort survey in 2100 patients with painful diabetic neuropathy and postherpetic neuralgia: differences in demographic data and sensory symptoms. Pain. 2009; 146:34-40.

2. Tesfaye S, Boulton AJ, Dickenson AH. Mechanisms and management of diabetic painful distal symmetrical polyneuropathy. Diabetes Care. 2013; 36:2456-2465.

3. Dellon AL. Treatment of symptomatic diabetic neuropathy by surgical decompression of multiple peripheral nerves. Plast Reconstr Surg. 1992; 89:689-697; discussion 698-689.

4. Upton AR, McComas AJ. The double crush in nerve entrapment syndromes. Lancet. 1973; 2:359-362.

5. Dellon AL, Muse VL, Scott ND, Akre T, Anderson SR, Barret SL, Biddinger KR, Bregman PJ, Bullard BP, Dauphinee DM, DeJesus JM, DeJesus RA, Ducic I, et al. A positive Tinel sign as predictor of pain relief or sensory recovery after decompression of chronic tibial nerve compression in patients with diabetic neuropathy. J Reconstr Microsurg. 2012; 28:235-240.

6. Baravarian B. Surgical decompression for painful diabetic peripheral nerve compression and neuropathy: a comprehensive approach to a potential surgical problem. Clin Podiatr Med Surg. 2006; 23:621-635.

7. Khan GM, Chen SR, Pan HL. Role of primary afferent nerves in allodynia caused by diabetic neuropathy in rats. Neuroscience. 2002; 114:291-299.

8. Sandkuhler J. Models and mechanisms of hyperalgesia and allodynia. Physiol Rev. 2009; 89:707-758.

9. Chen SR, Pan HL. Hypersensitivity of spinothalamic tract neurons associated with diabetic neuropathic pain in rats. $\mathrm{J}$ Neurophysiol. 2002; 87:2726-2733.

10. Xu ZZ, Kim YH, Bang S, Zhang Y, Berta T, Wang F, Oh $\mathrm{SB}, \mathrm{Ji} \mathrm{RR}$. Inhibition of mechanical allodynia in neuropathic pain by TLR5-mediated A-fiber blockade. Nat Med. 2015; 21:1326-1331.

11. Melzack R, Wall PD. Pain mechanisms: a new theory. Science. 1965; 150:971-979.

12. Wall PD. The gate control theory of pain mechanisms. A re-examination and re-statement. Brain. 1978; 101:1-18.

13. Todd AJ. Neuronal circuitry for pain processing in the dorsal horn. Nat Rev Neurosci. 2010; 11:823-836.

14. Chu DC, Albin RL, Young AB, Penney JB. Distribution and kinetics of GABAB binding sites in rat central nervous system: a quantitative autoradiographic study. Neuroscience. 1990; 34:341-357.

15. Wang XL, Zhang HM, Li DP, Chen SR, Pan HL. Dynamic regulation of glycinergic input to spinal dorsal horn neurones by muscarinic receptor subtypes in rats. J Physiol. 2006; 571:403-413.

16. Li DP, Chen SR, Pan YZ, Levey AI, Pan HL. Role of presynaptic muscarinic and $\mathrm{GABA}(\mathrm{B})$ receptors in spinal glutamate release and cholinergic analgesia in rats. J Physiol. 2002; 543:807-818.

17. Iyadomi M, Iyadomi I, Kumamoto E, Tomokuni K, Yoshimura M. Presynaptic inhibition by baclofen of miniature EPSCs and IPSCs in substantia gelatinosa neurons of the adult rat spinal dorsal horn. Pain. 2000; 85:385-393.

18. Wang XL, Zhang Q, Zhang YZ, Liu YT, Dong R, Wang QJ, Guo YX. Downregulation of GABAB receptors in the spinal cord dorsal horn in diabetic neuropathy. Neurosci Lett. 2011; 490:112-115.

19. Lee D, Dauphinee DM. Morphological and functional changes in the diabetic peripheral nerve: using diagnostic ultrasound and neurosensory testing to select candidates for nerve decompression. J Am Podiatr Med Assoc. 2005; 95:433-437.

20. Dellon AL, Mackinnon SE, Seiler WAt. Susceptibility of the diabetic nerve to chronic compression. Ann Plast Surg. 1988; 20:117-119. 
21. Jakobsen J. Peripheral nerves in early experimental diabetes: expansion of the endoneurial space as a cause of increased water content. Diabetologia. 1978; 14:113-119.

22. Barrett SL, Dellon AL, Fleischli J, Gould JS, Wang C. Metabolic and compressive neuropathy. Foot Ankle Spec. 2010; 3:132-139.

23. Battista AF, Alban E. Effect of graded ligature compression on nerve conduction. Exp Neurol. 1983; 80:186-194.

24. Basbaum AI, Bautista DM, Scherrer G, Julius D. Cellular and molecular mechanisms of pain. Cell. 2009; 139:267-284.

25. Malik RA. Pathology of human diabetic neuropathy. Handb Clin Neurol. 2014; 126:249-259.

26. Karagoz H, Yuksel F, Ulkur E, Celikoz B. Early and late results of nerve decompression procedures in diabetic neuropathy: a series from Turkiye. J Reconst Microsurg. 2008; 24:95-101.

27. Aszmann O, Tassler PL, Dellon AL. Changing the natural history of diabetic neuropathy: incidence of ulcer/ amputation in the contralateral limb of patients with a unilateral nerve decompression procedure. Ann Plast Surg. 2004; 53:517-522.

28. Siemionow M, Alghoul M, Molski M, Agaoglu G. Clinical outcome of peripheral nerve decompression in diabetic and nondiabetic peripheral neuropathy. Ann Plast Surg. 2006; 57:385-390

29. Ossipov MH, Bian D, Malan TP Jr, Lai J, Porreca F. Lack of involvement of capsaicin-sensitive primary afferents in nerve-ligation injury induced tactile allodynia in rats. Pain. 1999; 79:127-133.

30. Herder C, Kannenberg JM, Huth C, Carstensen-Kirberg M, Rathmann W, Koenig W, Heier M, Puttgen S, Thorand B, Peters A, Roden M, Meisinger C, Ziegler D. Proinflammatory cytokines predict the incidence and progression of distal sensorimotor polyneuropathy: KORA F4/FF4 study. Diabetes Care. 2017; 40:569-576.

31. Calcutt NA. Potential mechanisms of neuropathic pain in diabetes. Int Rev Neurobiol. 2002; 50:205-228.

32. Jensen TS, Finnerup NB. Allodynia and hyperalgesia in neuropathic pain: clinical manifestations and mechanisms. Lancet Neurol. 2014; 13:924-935.

33. Obrosova IG. Diabetic painful and insensate neuropathy: pathogenesis and potential treatments. Neurotherapeutics. 2009; 6:638-647.

34. Veves A, Backonja M, Malik RA. Painful diabetic neuropathy: epidemiology, natural history, early diagnosis, and treatment options. Pain Med. 2008; 9:660-674.

35. Veves A, Manes C, Murray HJ, Young MJ, Boulton AJ. Painful neuropathy and foot ulceration in diabetic patients. Diabetes Care. 1993; 16:1187-1189.

36. Dyck PJ, Dyck PJ, Larson TS, O’Brien PC, Velosa JA. Patterns of quantitative sensation testing of hypoesthesia and hyperalgesia are predictive of diabetic polyneuropathy: a study of three cohorts. Nerve growth factor study group. Diabetes Care. 2000; 23:510-517.

37. Otto M, Bak S, Bach FW, Jensen TS, Sindrup SH. Pain phenomena and possible mechanisms in patients with painful polyneuropathy. Pain. 2003; 101:187-192.

38. Eldor R, Raz I, Ben Yehuda A, Boulton AJ. New and experimental approaches to treatment of diabetic foot ulcers: a comprehensive review of emerging treatment strategies. Diabet Med. 2004; 21:1161-1173.

39. Yagihashi S, Yamagishi S, Wada R. Pathology and pathogenetic mechanisms of diabetic neuropathy: correlation with clinical signs and symptoms. Diabet Res Clin Pract. 2007; 77:S184-189.

40. Calcutt NA, Freshwater JD, Mizisin AP. Prevention of sensory disorders in diabetic Sprague-Dawley rats by aldose reductase inhibition or treatment with ciliary neurotrophic factor. Diabetologia. 2004; 47:718-724.

41. Cameron NE, Gibson TM, Nangle MR, Cotter MA. Inhibitors of advanced glycation end product formation and neurovascular dysfunction in experimental diabetes. Ann N Y Acad Sci. 2005; 1043:784-792.

42. Cameron NE, Cotter MA. Effects of protein kinase Cbeta inhibition on neurovascular dysfunction in diabetic rats: interaction with oxidative stress and essential fatty acid dysmetabolism. Diabetes Metab Res Rev. 2002; 18:315-323.

43. Ilnytska O, Lyzogubov VV, Stevens MJ, Drel VR, Mashtalir N, Pacher P, Yorek MA, Obrosova IG. Poly(ADP-ribose) polymerase inhibition alleviates experimental diabetic sensory neuropathy. Diabetes. 2006; 55:1686-1694.

44. Li F, Drel VR, Szabo C, Stevens MJ, Obrosova IG. Lowdose poly(ADP-ribose) polymerase inhibitor-containing combination therapies reverse early peripheral diabetic neuropathy. Diabetes. 2005; 54:1514-1522.

45. Kamiya H, Zhang W, Sima AA. C-peptide prevents nociceptive sensory neuropathy in type 1 diabetes. Ann Neurol. 2004; 56:827-835.

46. Obrosova IG, Xu W, Lyzogubov VV, Ilnytska O, Mashtalir N, Vareniuk I, Pavlov IA, Zhang J, Slusher B, Drel VR. PARP inhibition or gene deficiency counteracts intraepidermal nerve fiber loss and neuropathic pain in advanced diabetic neuropathy. Free Radic Biol Med. 2008; 44:972-981.

47. Pertovaara A, Wei H, Kalmari J, Ruotsalainen M. Pain behavior and response properties of spinal dorsal horn neurons following experimental diabetic neuropathy in the rat: modulation by nitecapone, a COMT inhibitor with antioxidant properties. Exp Neurol. 2001; 167:425-434.

48. Vareniuk I, Pavlov IA, Obrosova IG. Inducible nitric oxide synthase gene deficiency counteracts multiple manifestations of peripheral neuropathy in a streptozotocininduced mouse model of diabetes. Diabetologia. 2008; 51:2126-2133. 
49. Christianson JA, Ryals JM, Johnson MS, Dobrowsky RT, Wright DE. Neurotrophic modulation of myelinated cutaneous innervation and mechanical sensory loss in diabetic mice. Neuroscience. 2007; 145:303-313.

50. Drel VR, Pacher P, Vareniuk I, Pavlov IA, Ilnytska O, Lyzogubov VV, Bell SR, Groves JT, Obrosova IG. Evaluation of the peroxynitrite decomposition catalyst $\mathrm{Fe}(\mathrm{III})$ tetra-mesitylporphyrin octasulfonate on peripheral neuropathy in a mouse model of type 1 diabetes. Int J Mol Med. 2007; 20:783-792.

51. Jaffey PB, Gelman BB. Increased vulnerability to demyelination in streptozotocin diabetic rats. J Comp Neurol. 1996; 373:55-61.

52. Yagihashi S, Kamijo M, Watanabe K. Reduced myelinated fiber size correlates with loss of axonal neurofilaments in peripheral nerve of chronically streptozotocin diabetic rats. Am J Pathol. 1990; 136:1365-1373.

53. Lennertz RC, Medler KA, Bain JL, Wright DE, Stucky CL. Impaired sensory nerve function and axon morphology in mice with diabetic neuropathy. J Neurophysiol. 2011; 106:905-914.

54. Ma W, Eisenach JC. Chronic constriction injury of sciatic nerve induces the up-regulation of descending inhibitory noradrenergic innervation to the lumbar dorsal horn of mice. Brain Res. 2003; 970:110-118.

55. Jiang BC, Sun WX, He LN, Cao DL, Zhang ZJ, Gao YJ. Identification of IncRNA expression profile in the spinal cord of mice following spinal nerve ligation-induced neuropathic pain. Mol Pain. 2015; 11:43.

56. Richner M, Bjerrum OJ, Nykjaer A, Vaegter CB. The spared nerve injury (SNI) model of induced mechanical allodynia in mice. J Vis Exp. 2011; 5:3092.

57. Costigan M, Scholz J, Woolf CJ. Neuropathic pain: a maladaptive response of the nervous system to damage. Ann Rev Neurosci. 2009; 32:1-32.
58. Baron R, Binder A, Wasner G. Neuropathic pain: diagnosis, pathophysiological mechanisms, and treatment. Lancet Neurol. 2010; 9:807-819.

59. Spallone V, Greco C. Painful and painless diabetic neuropathy: one disease or two? Curr Diabet Rep. 2013; 13:533-549.

60. Malik RA, Veves A, Walker D, Siddique I, Lye RH, Schady W, Boulton AJ. Sural nerve fibre pathology in diabetic patients with mild neuropathy: relationship to pain, quantitative sensory testing and peripheral nerve electrophysiology. Acta Neuropathol. 2001; 101:367-374.

61. Britland ST, Young RJ, Sharma AK, Clarke BF. Acute and remitting painful diabetic polyneuropathy: a comparison of peripheral nerve fibre pathology. Pain. 1992; 48:361-370.

62. Dixon WJ. Efficient analysis of experimental observations. Ann Rev Pharmacol Toxicol. 1980; 20:441-462.

63. Chaplan SR, Bach FW, Pogrel JW, Chung JM, Yaksh TL. Quantitative assessment of tactile allodynia in the rat paw. J Neurosci Methods. 1994; 53:55-63.

64. Chen SR, Pan HL. Spinal endogenous acetylcholine contributes to the analgesic effect of systemic morphine in rats. Anesthesiology. 2001; 95:525-530.

65. Ochoa J. Recognition of unmyelinated fiber disease: morphologic criteria. Muscle Nerve. 1978; 1:375-387.

66. Lawson SN, Waddell PJ. Soma neurofilament immunoreactivity is related to cell size and fibre conduction velocity in rat primary sensory neurons. J Physiol. 1991; 435:41-63.

67. McCoy ES, Taylor-Blake B, Street SE, Pribisko AL, Zheng J, Zylka MJ. Peptidergic CGRPalpha primary sensory neurons encode heat and itch and tonically suppress sensitivity to cold. Neuron. 2013; 78:138-151. 